\title{
Correction to: Influence of tumor extent on central lymph node metastasis in solitary papillary thyroid microcarcinomas: a retrospective study of 1092 patients
}

Xingjie Yin, Chunping Liu, Yawen Guo, Xiaoyu Li, Na Shen, Xiangwang Zhao, Pan Yu, Shan Wang and Zeming Liu

\section{Erratum}

After publication of the original article [1] it was noted that the affiliation address had been incorrectly submitted by the authors.

The address affiliation address listed currently reads "Department of Breast and Thyroid Surgery, Union Hospital, Tongji Medical College, Huazhong University of Science and Technology, Number 1277, Jiefang Road, Wuhan, Hubei Province, China".

At present the correct address is "Department of Breast and Thyroid Surgery, Union Hospital, Tongji Medical College, Huazhong University of Science and Technology, Wuhan 430022, China".

This error had since been acknowledged and corrected in this correction article. The authors also wish to apologize for any inconvenience caused.

Published online: 17 October 2017

\section{Reference}

1. Yin X, Liu C, Guo Y, Li X, Shen N, Zhao X, Yu P, Wang S, Liu Z. Influence of tumor extent on central lymph node metastasis in solitary papillary thyroid microcarcinomas: a retrospective study of 1092 patients. World J Surg Oncol. 2017;15(1):133.

\footnotetext{
*Correspondence: Icp191@163.com

The online version of the original article can be found under doi:10.1186/ s12957-017-1202-8

Department of Breast and Thyroid Surgery, Union Hospital, Tongji Medical College, Huazhong University of Science and Technology, Wuhan 430022, China
} 